\title{
Plasmonic excitations in noble metals: The case of $\mathrm{Ag}$
}

\author{
M. A. Cazalilla* and J. S. Dolado \\ Materialen Fisika Saila, Euskal Herriko Unibertsitatea, 1072 PostaKutxatila, 20080 Donostia, Spain
}

\author{
A. Rubio \\ Departamento de Física Teórica, Universidad de Valladolid, E-47011 Valladolid, Spain \\ and Donostia International Physics Center, Donostia, Spain
}

P. M. Echenique

Materialen Fisika Saila, Euskal Herriko Unibertsitatea, 1072 Postakutxatila, 20080 Donostia, Spain and Centro Mixto CSIC-UPV/EHU, Spain

(Received 9 July 1999; revised manuscript received 5 November 1999)

\begin{abstract}
The delicate interplay between plasmonic excitations and interband transitions in noble metals is described by means of $a b$ initio calculations and a simple model in which the conduction electron plasmon is coupled to the continuum of electron-hole pairs. Band-structure effects, especially the energy at which the excitation of the $d$-like bands takes place, determine the existence of a threshold plasmonic mode, which manifests itself in $\mathrm{Ag}$ as a sharp resonance experimentally observed at $3.8 \mathrm{eV}$. However, such a resonance does not appear in the spectra of the other noble metals. Here this different behavior is also analyzed, and an explanation is provided.
\end{abstract}

\section{INTRODUCTION}

In a recent letter, ${ }^{1} \mathrm{Ku}$ and Eguiluz showed that bandstructure effects are responsible for the positive dispersion of the plasmon line width in K. Such a behavior was previously found by vom Felde, Sprösser-Prou, and Fink ${ }^{2}$ in electronenergy-loss experiments. They interpreted the result as due to short-range electron-electron correlations beyond the random-phase approximation (RPA). However, $\mathrm{Ku}$ and Eguiluz explained the result within the RPA in terms of the extra plasmon decay channels provided by a manifold of unoccupied $d$-symmetry bands. The effect of these bands is overlooked when treating $\mathrm{K}$ as a homogeneous electron system in the jellium model. Therefore, band-structure effects turn out to be decisive in determining not only the existence of interband transitions, but also the properties of plasmonic excitations. Indeed, $a b$ initio calculations of the dynamical response have properly described the experimental plasmon dispersion in a variety of simple metals. ${ }^{3-6}$ On the other hand, the jellium model still provides a qualitative, though not accurate, description of the energy-loss spectrum of these systems in the long-wavelength limit.

The energy-loss spectrum can be obtained either by performing electron-energy-loss spectroscopy ${ }^{7}$ or inelastic scattering of x-ray photons, ${ }^{8}$ or indirectly by means of optical measurements. ${ }^{9}$ In noble metals this spectrum has a complicated structure, which bears little resemblance to that of simple metals. It is known ${ }^{10}$ that such a structure has to do with the existence of a manifold of $d$-like bands a few eV below the Fermi level. These lead to important deviations from even the crudest predictions for an homogeneous electron liquid. Thus, whereas for Ag a narrow resonance is observed at an energy $\approx 3.8 \mathrm{eV}$, no well-defined plasmonic excitations appear in the spectra of $\mathrm{Cu}$ and $\mathrm{Au}$.

The purpose of this paper is to investigate the structure of the energy-loss spectrum in the long-wavelength limit, where plasmonic excitations are expected to be important. In particular, we have focused on Ag for our study, but some of our conclusions also apply to $\mathrm{Cu}$ and $\mathrm{Au}$, and could be extended to similar metallic compounds with fully occupied $d$-like bands close to the Fermi level. The spectrum of $\mathrm{Cu}$ was studied from first principles in a previous work. ${ }^{11}$ In the present work, we show that the structure in the energy-loss spectrum of $\mathrm{Ag}$ up to $\sim 10 \mathrm{eV}$ can be understood in terms of a simple model. In particular, we are concerned with the role played by the relative values of the Drude plasma frequency and the threshold energy for excitation of the $d$-like bands. After clarifying the relevant elements for the existence of a plasmonic excitation around the interband excitation threshold, numerical experiments in an $a b$ initio framework for calculating the dielectric matrix of $\mathrm{Ag}$ are performed to confirm this picture. Furthermore, a comparison of the $a b$ initio results with experimental data displays good agreement for excitations well above the threshold, being able to reproduce a structure in the loss spectrum up to $\approx 30 \mathrm{eV}$. An underestimate of the threshold energy in local-density calculations also shows up in our calculations, yielding a lower plasma frequency than the experiments.

The question of how band structure alters the properties of plasmons in metals was previously addressed by a number of authors. Wilson ${ }^{12}$ studied the shift in the Drude plasma frequency due to the presence of an optical-absorption band that lies above (below) the free-electron plasma frequency. He found that the plasma frequency is shifted downwards (upwards) by the effect of the absorption band. More recently, Sturm ${ }^{13}$ studied, within the RPA, the corrections to the dielectric function of an homogeneous electron gas due to a weak lattice potential. He showed that the lattice potential can provide the necessary momentum for a plasmon to decay into an electron-hole pair below the Landau cutoff. ${ }^{14}$ 
In other words, when taking the lattice structure into account by folding the free-electron bands into the first Brillouin zone (BZ), a plasmonic excitation can decay by producing an interband transition. As a consequence, even in the RPA, plasmons acquire a finite lifetime. Core polarization effects on the plasma frequency were investigated by Zaremba and Sturm. ${ }^{15}$ They treated the core polarizability as a superposition of the polarizability of the isolated lattice ions. The polarization of core electrons lowers the plasma frequency from its Drude value, bringing it closer to the experimental value.

The behavior of noble metals was considered by Ehrenreich and Phillipp. ${ }^{10}$ These authors used the data obtained in experimental measurements of the reflectivity from $\mathrm{Cu}$ and $\mathrm{Ag}^{16}$ to obtain the optical and energy-loss spectra. They separated the Drude and interband contributions to the optical response, thus shedding light on the reason why the energy of the plasma resonance in Ag is shifted down in energy. In a later work, Cooper, Ehrenreich and Phillipp ${ }^{17}$ extended this analysis to Au. They touched upon the question of assigning interband transitions to the most relevant features observed in the optical spectrum. To further clarify this point, Mueller and Phillips ${ }^{18}$ performed, within the RPA and based on band structure calculations by Burdick, ${ }^{19}$ a numerical calculation of the imaginary part of the interband contribution to the optical response of $\mathrm{Cu}$. Thus they could give a more correct identification of the interband transitions that contribute to the optical spectrum at a given energy. Their interpretation is consistent with the optical data obtained in a number of reflectivity measurements from $\mathrm{Ag}$ and Ag alloys. ${ }^{20,21}$

Recently, the dielectric matrix as well as the energy-loss spectrum have been evaluated numerically within a firstprinciples framework. To mention a few examples of theses studies, in addition to the work by $\mathrm{Ku}$ and Eguiluz ${ }^{1}$ mentioned above, Quong and Eguiluz ${ }^{3}$ thus investigated the anisotropy in the plasmon dispersion of aluminum. ${ }^{22}$ Aryasetiawan and Karlsson ${ }^{4}$ studied the excitations in the energyloss spectra of alkali metals. The negative dispersion of bulk plasmons in Cs was investigated using these methods by Aryasetiawan and Karlsson ${ }^{4}$ and Fleszar et al. ${ }^{5}$ In general, specific features of the band structure such as the presence of flat $d$-like bands and gaps at the Brillouin-zone boundaries are needed to describe in detail the experimental energy-loss spectra in simple ${ }^{6,23-27}$ and noble metals. ${ }^{11}$ In the case of noble metals, the energy-loss function of $\mathrm{Cu}$ was evaluated from first principles by Campillo, Rubio, and Pitarke. ${ }^{11}$ An accurate description of the energy-loss spectrum of $\mathrm{Cu}$ is thus attained once the fully occupied $d$-like bands are considered as valence bands. In this work, we obtain the energyloss spectrum of Ag using the same ab initio techniques, but this time we focus on understanding the plasmonic excitations in noble metals. The appearance of a plasma resonance at $\approx 3.8 \mathrm{eV}$ in $\mathrm{Ag}$ can also be qualitatively understood with the help of a simple model, which provides a perspective on how a threshold plasmonic mode can appear, and shows the parameters controlling the existence of this type of excitations.

The outline of the paper is as follows. In Sec. II we discuss a model which provides a qualitative explanation for some features in the energy-loss spectrum up to $\sim 10 \mathrm{eV}$. The details of the $a b$ initio computation of the spectrum can be found in Sec. III. A description and discussion of the results is given in Sec. IV. Finally, the main conclusions of the present paper are summarized in Sec. V. Atomic units $\left(\hbar=e^{2}=m_{e}=1\right)$ are used in all mathematical expressions.

\section{A SIMPLE MODEL}

In order to estimate the plasma frequency in $\mathrm{Ag}$, we can try to use the classical expression ${ }^{28} \omega_{p}=\sqrt{4 \pi n}$. If we naively set $n=n_{c}$, i.e., the density of those electrons coming from the atomic $5 s$ orbital, we find $\omega_{p}^{c}=8.98 \mathrm{eV}$. This estimate sets the energy scale for a collective excitation involving these electrons, which are supposed to be the least tightly bound and to populate the states high in the conduction band. However, the value thus obtained is in clear disagreement with the experimental observation of a narrow plasma resonance at $3.8 \mathrm{eV}$. Including the ten electrons from the atomic $4 d$ orbitals worsens the estimate, leading to a value for $\omega_{p}$ of $32.6 \mathrm{eV}$, which sets the energy scale for a collective excitation involving the electrons in the $d$-like and conduction $s p$-like bands. In this section, we shall develop a model that applies to the lower-energy scale set by $\omega_{p}^{c}$, and illustrates how band-structure effects can modify the energy-loss spectrum of $\mathrm{Ag}$ in the long-wavelength limit so that it does not show the same structure as simple metals.

The usual method to determine the position of plasma resonances in the long-wavelength energy-loss spectrum goes as follows. Disregarding crystal local-field effects, (well-defined) plasmonic excitations correspond to zeroes in the macroscopic dielectric function. For $|\mathbf{q}|=0$, we have

$$
\epsilon(\Omega)=0
$$

for some complex $\Omega$ with a small imaginary part. Using the Sellmeyer-Drude expression ${ }^{29}$ for $\epsilon(\omega)$, and neglecting damping effects,

$$
\epsilon(\omega)=1-\frac{\left(\omega_{p}^{D}\right)^{2}}{\omega^{2}}+\delta \epsilon^{(i b)}(\omega),
$$

where last term corresponds to the interband contribution:

$$
\delta \epsilon^{(i b)}(\omega)=\sum_{\omega_{n}>0} \frac{f_{n}}{\omega_{n}^{2}-\omega^{2}} .
$$

The condition in Eq. (1) can be written as

$$
\Omega^{2}-\left(\omega_{p}^{D}\right)^{2}+\Omega^{2} \delta \epsilon^{(i b)}(\Omega)=0 .
$$

The oscillator strength, $f_{n}$ in Eq. (3), is a measure of the effective number of electrons that participate in the excitation of a given mode $n$, with energy $\omega_{n}$. The dominant contribution at frequencies well below $\omega_{p}^{c}$ comes from the excitation of conduction electrons. The Drude plasma frequency ${ }^{10} \omega_{p}^{D}=9.2 \mathrm{eV}$ is slightly higher than $\omega_{p}^{c}$. The ratio of the two plasma frequencies is usually expressed in terms of the "optical" mass, $m_{\text {opt }}$ (recall that $m_{e}=1$ in atomic units): 


$$
\left(\frac{\omega_{p}^{c}}{\omega_{p}^{D}}\right)^{2}=m_{o p t}=0.95
$$

for $\mathrm{Ag}$ and $m_{\text {opt }}=1.35$ for $\mathrm{Cu} .{ }^{10}$ In the RPA, $m_{\text {opt }}$ corresponds to the effective mass of electrons averaged over the occupied part of the conduction band.

Equation (4) can be read in the following way. We assume that the term $\Omega^{2} \delta \epsilon^{(i b)}(\Omega)$ plays the role of a "selfenergy" for the Drude plasmon. In simple metals, this term is usually small and varies slowly with $\omega$. This leads to a small shift of the Drude frequency. The plasma frequency also acquires a small imaginary part, which accounts for the fact that the plasmon state is degenerate in energy with other configurations of the system involving one or several electrons excited from one band to another. This idea can easily be realized in a model in which the plasmon is viewed as a boson coupled to an energy continuum. In an independentelectron picture, the continuum corresponds to the spectrum of electron-hole pairs. A simple model Hamiltonian that can be set up to describe this situation is

$$
\begin{aligned}
H= & \sum_{\mathbf{q}} \omega_{p}^{D} b_{\mathbf{q}}^{\dagger} b_{\mathbf{q}}+\sum_{k, \mathbf{q}} \omega_{k, \mathbf{q}} m_{k, \mathbf{q}}^{\dagger} m_{k, \mathbf{q}} \\
& +\sum_{\mathbf{q}, k} g_{k, \mathbf{q}}\left(b_{\mathbf{q}}+b_{-\mathbf{q}}^{\dagger}\right)\left(m_{k, \mathbf{q}}^{\dagger}+m_{-k,-\mathbf{q}}\right) .
\end{aligned}
$$

In this model, plasmons are presented as a field of oscillators carrying momentum q. Thus, $b_{\mathbf{q}}^{\dagger}$ and $b_{\mathbf{q}}$ are plasmon creation and annihilation operators, respectively. To keep the model as simple as possible while retaining all the important physical effects, the energy continuum is also represented by a set of harmonic oscillators whose quanta are created and annihilated by the operators $m_{k, \mathbf{q}}^{\dagger}$ and $m_{k, \mathbf{q}}$. The label $k$ stands for the additional quantum numbers (spin, relative momentum, band indices, etc.) carried by a given continuum mode. The operator $m_{-k,-\mathbf{q}}\left(m_{-k,-\mathbf{q}}^{\dagger}\right)$ anhilates (creates) a continuum mode with all the momentum quantum numbers reversed. The assumed interaction between plasmons and the continuum conserves the momentum q. Terms connecting plasmons and continuum modes with momentum differing by a finite reciprocal-lattice vector are therefore neglected. This approximation amounts to disregarding crystal local-field corrections, which in general is a good approximation for metals. Indeed, the $a b$ initio calculations for $\mathrm{Cu}^{11}$ and $\mathrm{Ag}$ (see below) show that crystal local-field effects at small $\mathbf{q}$ amount at most to a ten percent correction in the peak intensity.

The above model [Eq. (6)] can be solved exactly. ${ }^{30,31}$ Indeed, it is related to the models introduced by Fano ${ }^{32}$ and Anderson $^{33}$ to study the coupling of a discrete state to a continuum. More precisely, the present model can be thought of as an extension of Gadzuk's work ${ }^{30}$ for a localized vibrational mode in an electron liquid. Thus the energies of the plasmonic modes are given by the solutions of the equation

$$
\Omega^{2}-\left(\omega_{p}^{D}\right)^{2}+2 \omega_{p}^{D} M(\mathbf{q}, \Omega)=0,
$$

where $M(\mathbf{q}, \omega)$ is the plasmon self-energy. The real part of it can be obtained from the imaginary part

$$
\operatorname{Im} M(\mathbf{q}, \omega)=\pi \sum_{k}\left|g_{k, \mathbf{q}}\right|^{2}\left[\delta\left(\omega-\omega_{k, \mathbf{q}}\right)-\delta\left(\omega+\omega_{k, \mathbf{q}}\right)\right],
$$

by Kramers-Kroning

$$
\operatorname{Re} M(\mathbf{q}, \omega)=\mathrm{P} \int \frac{\mathrm{d} \omega}{\pi} \frac{\operatorname{Im} M(\mathbf{q}, \omega)}{\omega^{\prime}-\omega}
$$

Working in analogy with Eq. (4), we could tentatively identify

$$
M(|\mathbf{q}| \rightarrow 0, \omega) \approx \frac{\omega^{2}}{2 \omega_{p}^{D}} \delta \epsilon^{(i b)}(\omega)
$$

for $\omega \sim \omega_{p}^{c}$. For arbitrary $\omega$, however, it is better to look at $\operatorname{Im} M(\mathbf{q}, \omega)$ as a weighted density of the continuum modes [cf. Eq. (8)], so that the resulting real part of $M(\mathbf{q}, \omega)$ accounts for the polarization effects associated with the propagation of a Drude plasmon. Thus it follows from Eqs. (7)(9) that if $\omega_{p}^{D}$ lies above (below) the region where $\operatorname{Im} M(\mathbf{q}, \omega)$ is large [provided $\operatorname{Im} M\left(\mathbf{q}, \omega_{p}^{D}\right) / \omega_{p}^{D}$ is small], the position of plasma resonance will be shifted upwards (downwards) in energy. This result is in agreement with the abovementioned work by Wilson. ${ }^{12}$

However, when $\operatorname{Im} M\left(\mathbf{q}, \omega_{p}^{D}\right) / \omega_{p}^{D}$ is large, we will not observe a well-defined plasma resonance in the spectrum. This seems to be the case of noble metals due to the presence of a manifold of occupied $d$-like bands, which gives rise to a broad band of modes extending from the interband threshold energy $\omega_{T} \approx 3.9 \mathrm{eV}$ (for $\mathrm{Ag}$ and $2.1 \mathrm{eV}$ for $\mathrm{Cu}$ ), to well above the Drude plasma frequency $(9.2 \mathrm{eV}$ for $\mathrm{Ag}$ and $9.3 \mathrm{eV}$ for $\mathrm{Cu}$ ). If we assume that the onset of interband transitions in Ag takes place so sharply as to produce a discontinuity in the weighted density of continuum modes,

$$
\operatorname{Im} M(|\mathbf{q}| \rightarrow 0, \omega) \sim \theta\left(\omega_{T}-\omega\right),
$$

for $\omega \sim \omega_{T}$, then $\operatorname{Re} M(\mathbf{q} \mid \rightarrow 0, \omega)$ will develop a logarithmic singularity $\sim-\log \left(\omega-\omega_{T}\right)$ for $\omega<\omega_{T}$, leading to a dampingless solution of Eq. (7) just below the threshold $\omega_{T}$. This polaroniclike solution ${ }^{34}$ corresponds to hybrid plasmonic mode, in which the conduction electrons oscillate coherently without exciting the electrons in the $d$-like bands. These are just polarized by the electric field set up by the plasmon, thus lowering the value of plasma frequency. This point of view agrees well with more phenomenological approaches, ${ }^{35,36}$ which simply assume that the change in the plasma frequency can be accounted for by an effective dielectric function $\epsilon_{d}$ for the electrons in the $d$-like bands, so that $\omega_{p}^{*}$ $=\omega_{p}^{D} / \sqrt{\epsilon_{d}} \approx 3.8 \mathrm{eV}$.

Additionally, there is another complex solution of Eq. (7), for which the conduction electrons move as an overdamped oscillator, rapidly decaying into a continuum mode (i.e., producing an interband transition). For $|\mathbf{q}| \rightarrow 0$, this excitation will correspond to an optical interband transition. Two types of optical transitions may occur above the threshold, ${ }^{18}$ two examples of which have been indicated by arrows in Fig. 1, where we have plotted the calculated local-density- 


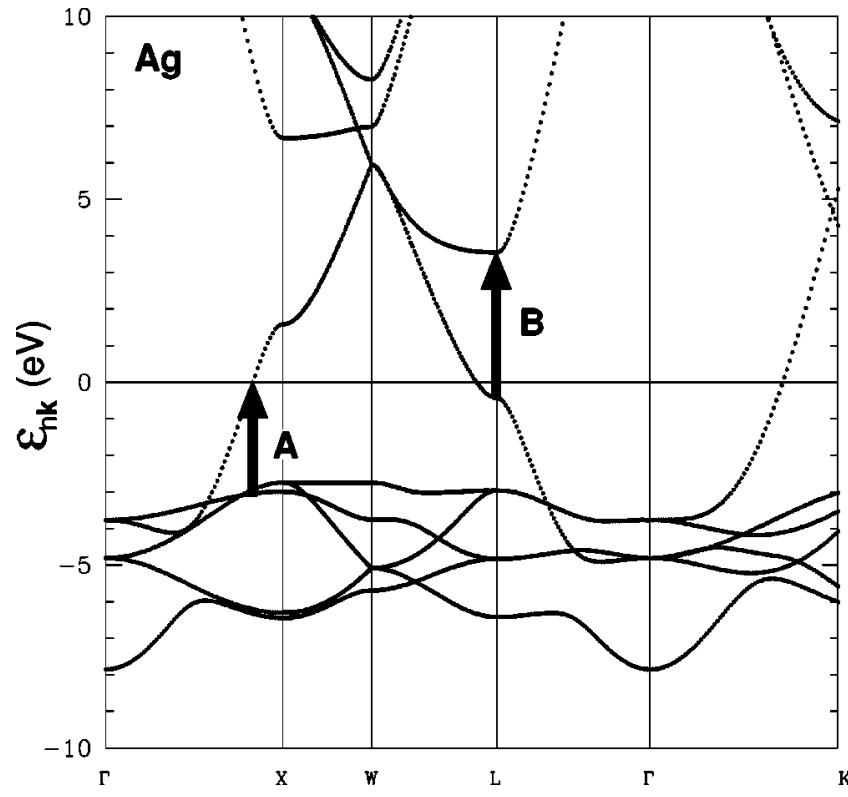

FIG. 1. Calculated LDA band structure of Ag. Arrows indicate two examples (A and $\mathrm{B}$ ) of the types of interband transitions that couple to the plasmonic excitations in the long-wavelength limit, for energies above the interband excitation threshold. The crystal structure is fcc, with a lattice parameter $a=4.09 \AA$. The zero energy corresponds to the Fermi level.

approximation (LDA) band structure of Ag. Type A corresponds to the excitation of an electron from one of the flat $d$-like bands to an unoccupied state in the conduction band or above. The other type (B), whose onset occurs at approximately the same energy in the three noble metals $(\approx 4.0 \mathrm{eV})$, corresponds to the excitation from an occupied state in the conduction band (near $L$ point in the BZ) to the flat part of an unoccupied band or above. This type of transition seems to be responsible for the damping of the plasmon when the $4 d$ electrons are included in the pseudocore, i.e., they are considered to be frozen. In Sec. IV, we shall explain this issue more in detail. These two types of transitions are important because they often involve flat bands, which may lead to van Hove singularities in the joint density of states.

In the case of $\mathrm{Ag}$, the onsets for the transitions of types $\mathrm{A}$ and B nearly overlap in energy. This produces a sharp interband onset and gives rise to the pronounced peak that Im $\epsilon(\omega)$ exhibits around $4 \mathrm{eV}$. From Eq. (10), it follows that $\operatorname{Im} M(|\mathbf{q}| \rightarrow 0, \omega)$ will also have a maximum at the same energy. The onset of the interband transitions at $\omega_{T}$ is not as sharp as suggested by Eq. (11). However, it still leads to a zero of $\Omega^{2}-\left(\omega_{p}^{D}\right)^{2}+2 \omega_{p}^{D} \operatorname{Re} M(|\mathbf{q}| \rightarrow 0, \Omega)$ for a real $\Omega$, which occurs below the threshold, where the density of continuum modes is very small. This point is illustrated by Fig. 2, which displays the graphical solution of Eq. (7). This provides an explanation for the existence of a threshold plasma resonance in $\mathrm{Ag}$. However, it remains to explain why a similar phenomenon does not take place in the spectra of $\mathrm{Cu}$ or $\mathrm{Au}$. The difference stems from the specific details of the band structure and, in particular, from the value of the threshold energy. In Fig. 3, we plot the graphical solution of Eq. (7) for the case of $\mathrm{Cu}$. The threshold for the interband excitation occurs this time at a lower energy $(2.1 \mathrm{eV})$, as the

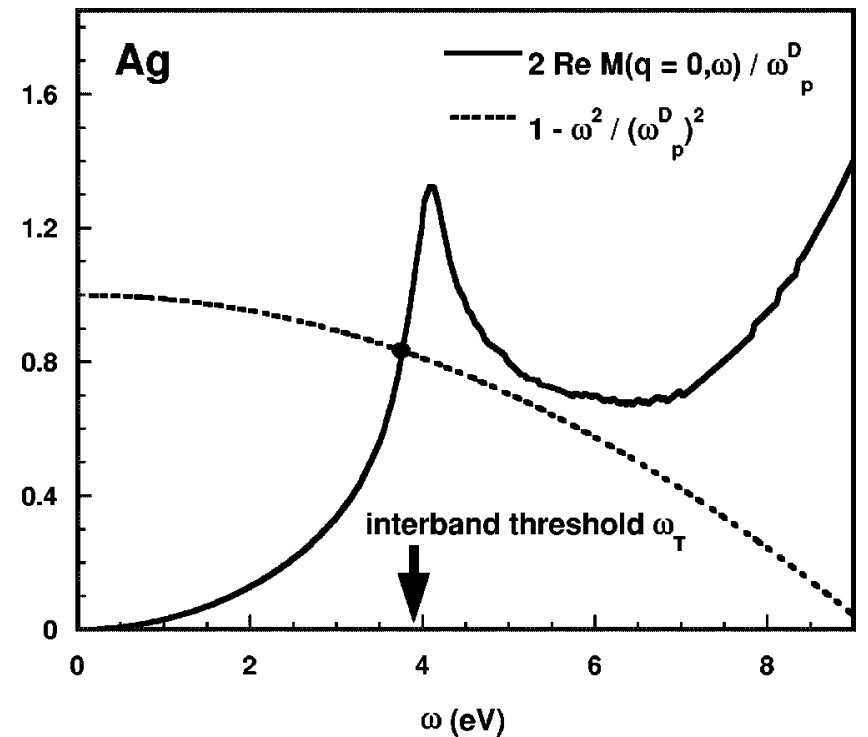

FIG. 2. Graphical solution of Eq. (7) for the threshold plasmonic modes in Ag. Data for $\delta \epsilon^{(i b)}(\omega)$ [i.e., for $\operatorname{Re} M(|\mathbf{q}| \rightarrow 0, \omega)$ ] are from Ref. 10. The continuous line cuts the dashed line at the point indicated by the black dot. This point lies just below the threshold, where the density of continuum modes is small. As a consequence, a narrow plasma resonance appears in the spectrum.

$d$-like bands lie closer to the Fermi level. A comparison of Figs. 2 and 3 shows the important role played by the value of the threshold energy in enhancing the maximum of $\operatorname{Im} M(|\mathbf{q}| \rightarrow 0, \omega) \quad$ [cf. Eq. (10)], and thus in $\operatorname{Re} M(|\mathbf{q}| \rightarrow 0, \omega)$. Therefore, for $\mathrm{Cu}$ there exists no zero of Eq. (7) close to the real axis, i.e., below the threshold, where this plasma mode would have a small density of continuum modes to decay. A similar conclusion can be drawn for the case of $\mathrm{Au}$.

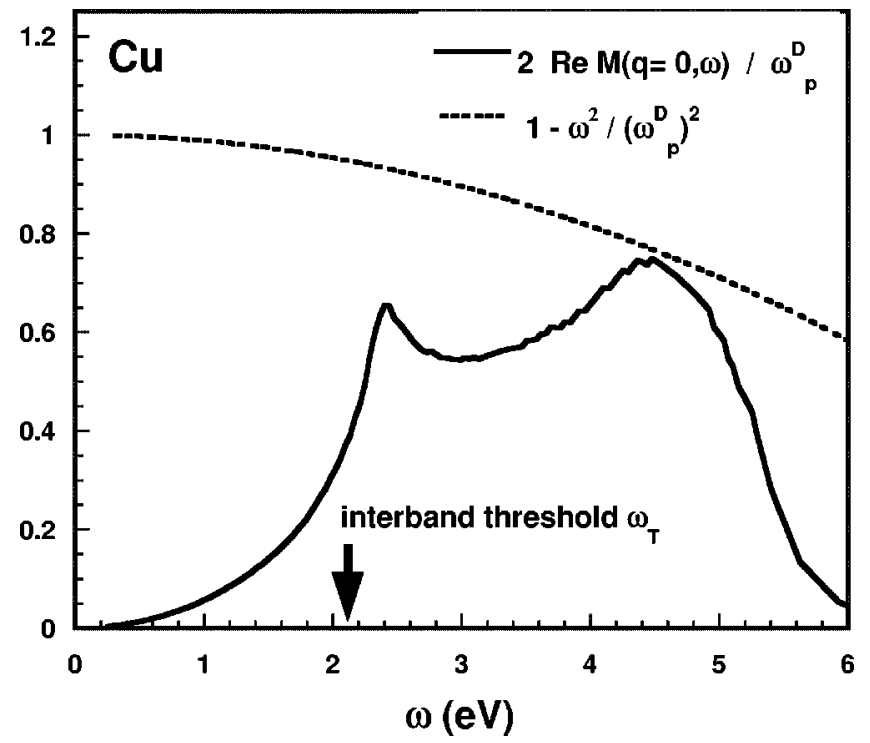

FIG. 3. Graphical solution of Eq. (7) for the threshold plasmonic modes in $\mathrm{Cu}$. Data for $\delta \epsilon^{(i b)}(\omega)$ [i.e., for $\operatorname{Re} M(|\mathbf{q}| \rightarrow 0, \omega)$ ] are from Ref. 10. The continuous line does not cut the dashed line, indicating that no threshold plasmonic modes exist in $\mathrm{Cu}$. A similar situation is expected to hold for Au. 
To sum up, interband transitions modify the original Drude plasmon, which turns out to be no longer a welldefined plasmonic excitation. Thus it shows up in the $\mathrm{Ag}$ spectrum as a broad peak with a maximum around $8 \mathrm{eV}$. Moreover, a combination of a high value for $\omega_{T}$ along with the sharp onset of interband transitions produces a narrow plasma resonance in Ag. In this respect, our conclusions agree with those of Ehrenreich and Phillipp. ${ }^{10}$ Furthermore, as the spectral weight of this polaroniclike feature in the spectrum is rather small, it is easily damped. This makes this plasmonic excitation very sensitive to changes in the band structure, impurities, or defects. This is consistent with the experimental evidence from alloying experiments,, ,20,21 which indicates that the plasmon in Ag is strongly damped by increasing the concentration of the other component in the alloy. Finally, it is worth pointing out that the existence of this resonance also depends on the value of the Drude plasma frequency. If the value of $\omega_{p}^{D}$ is increased, for example, by increasing the electron density in the system, the resonance will weaken and eventually disappear from the spectrum. This prediction is confirmed by our $a b$ initio results (shown below) for the energy-loss spectrum of Ag under externally applied hydrostatic pressure.

\section{DETAILS OF THE $A B I N I T I O$ CALCULATIONS}

By measuring the energy lost by electrons or x-ray photons in their interaction with matter, we can access the dynamical structure factor $S(\mathbf{q}, \omega)$ of a system. The fluctuationdissipation theorem ${ }^{37}$ then relates $S(\mathbf{q}, \omega)$ to the density correlation function $\chi\left(\mathbf{r}, \mathbf{r}^{\prime}, t-t^{\prime}\right)$, and time-dependent density-functional theory ${ }^{38}$ provides us with an a priori exact integral equation to compute the latter function. Usually, one has to resort to different approximation schemes in order to calculate $\chi$. In the case of simple ${ }^{1,3}$ and noble ${ }^{11}$ metals in the small- $|\mathbf{q}|$ limit, where collective excitations dominate the energy-loss spectrum, it has been found that the RPA provides a good description of the energy-loss spectrum. This approximation involves neglecting short-range exchange and correlation effects, and therefore $S(\mathbf{q}, \omega)$ can be obtained from the energy-loss function $\operatorname{Im}\left[-\epsilon_{\mathbf{0 0}}^{-1}(\mathbf{q}, \omega)\right]$, which follows from inverting the dielectric matrix,

$$
\epsilon_{\mathbf{G G}^{\prime}}(\mathbf{q}, \omega)=\delta_{\mathbf{G}^{\prime} \mathbf{G}^{\prime}}-v_{\mathbf{G}^{\prime}}(\mathbf{q}) \chi_{\mathbf{G G}^{\prime}}^{0}(\mathbf{q}, \omega),
$$

where $\mathbf{q}$ belongs to the BZ, and $\mathbf{G}$ and $\mathbf{G}^{\prime}$ are reciprocallattice vectors. We use the same notation and definitions as in Ref. 11. The function

$$
\begin{aligned}
\chi_{\mathbf{G G}^{\prime}}^{0}(\mathbf{q}, \omega)= & \frac{1}{V} \sum_{\mathbf{k} \in \mathrm{BZ}} \sum_{n, m} \frac{f\left(\varepsilon_{n \mathbf{k}}\right)-f\left(\varepsilon_{m \mathbf{k}+\mathbf{q}}\right)}{\omega+\varepsilon_{n \mathbf{k}}-\varepsilon_{m \mathbf{k}+\mathbf{q}}+i \eta} \\
& \times\left\langle n \mathbf{k}\left|e^{-i(\mathbf{q}+\mathbf{G}) \cdot \mathbf{r}}\right| m \mathbf{k}+\mathbf{q}\right\rangle \\
& \times\left\langle m \mathbf{k}+\mathbf{q}\left|e^{i\left(\mathbf{q}+\mathbf{G}^{\prime}\right) \cdot \mathbf{r}}\right| n \mathbf{k}\right\rangle,
\end{aligned}
$$

with $\eta \rightarrow 0^{+}$, is the density correlation function of a system of particles moving in the self-consistent LDA (Refs. 39 and 40) potential. The energies $\varepsilon_{n \mathbf{k}}$ and orbitals $|n \mathbf{k}\rangle$ are the corresponding eigenvalues and eigenvectors of the LDA
Hamiltonian. These states are Bloch states, and we have used a plane-wave basis to expand them. However, as electrons in the outer atomic $4 d$ orbitals play an important role in the response of noble metals, they must treated as a part of the valence electrons. The remaining core electrons are replaced by a frozen pseudocore using a scalar relativistic normconserving pseudopotential scheme. ${ }^{41}$ Therefore, these electrons will not respond to an external perturbation. This is not an important drawback as long as the excitation energy remains below the typical excitation energies of core electrons $\sim 100 \mathrm{eV}^{42}$

In order to further reduce the numerical damping $\eta$, we have also computed $\chi^{0}$ on the imaginary frequency axis by making the replacement $\omega+i \eta \rightarrow i \nu$ in Eq. (13). An analytic continuation to the real axis has been carried out by using a Padé approximant. ${ }^{43}$ This procedure allows us to use a numerical damping as small as $0.001 \mathrm{eV}$. This is to be compared with the $0.2-\mathrm{eV}$ value used in the real frequency calculations also presented in this work. However, we shall show the results of both types of calculations together, to indicate that one must be very careful with the analytic continuation procedure using Padé approximants. Thus the results obtained using this method tend to be smoother than what is obtained with a calculation with real frequencies and larger values of $\eta$. Thus it usually happens that some features of the spectrum, as obtained in a real frequency calculation, are completely missing from the analytically continued result (see Sec. IV). In fact, although we have used the Lentz algorithm ${ }^{44}$ to evaluate the corresponding continued fraction, we have been unable to ensure that the procedure converges by increasing the number of points over the same interval of $\nu$. When fitting the Padé approximant ${ }^{45}$ to the calculated values of $\epsilon_{\mathbf{0 0}}^{-1}(\mathbf{q}, i \nu)$, we have found that the results are unstable with respect to increasing the number of points. Thus we have obtained differences of the order of $10 \%$ when the number of points over the same interval was simply doubled. Finally, we have decided to use the modulus of the remainder of the truncated continued fraction to estimate the error, and always compare the outcome with a real frequency calculation prior to considering it as reliable.

\section{RESULTS}

In Fig. 1 we plot the band structure of $\mathrm{Ag}$ as calculated within the LDA. Two examples of the main types of interband transitions (A and B) which couple to plasmons at energies $\omega \sim \omega_{p}^{D}$ are indicated by arrows. In the LDA, we obtain a threshold energy for type-A transitions $\approx 3.0 \mathrm{eV}$, whereas experimentally it is found to be $\approx 4.0 \mathrm{eV} .{ }^{10}$ Since LDA eigenvalues enter the expression for $\chi^{0}$, this turns out to be the reason to underestimate the threshold frequency $\omega_{T}$, as shown in Figs. 4 and 5. An underestimate of this kind was already observed for $\mathrm{Cu}$, where $\omega_{T} \approx 1.5 \mathrm{eV}$, in contrast with the experimental value of $2.1 \mathrm{eV} .{ }^{10}$ The source of such an underestimate may be either a failure of the LDA or an indication that a frequency-dependent and perhaps nonlocal $K^{x c}$ may be required to account fully for the experimental value of $\omega_{T}$. In our opinion, however, it is related to a failure of the LDA, which may not be taking into account the strong correlations occurring among the electrons in the flat 


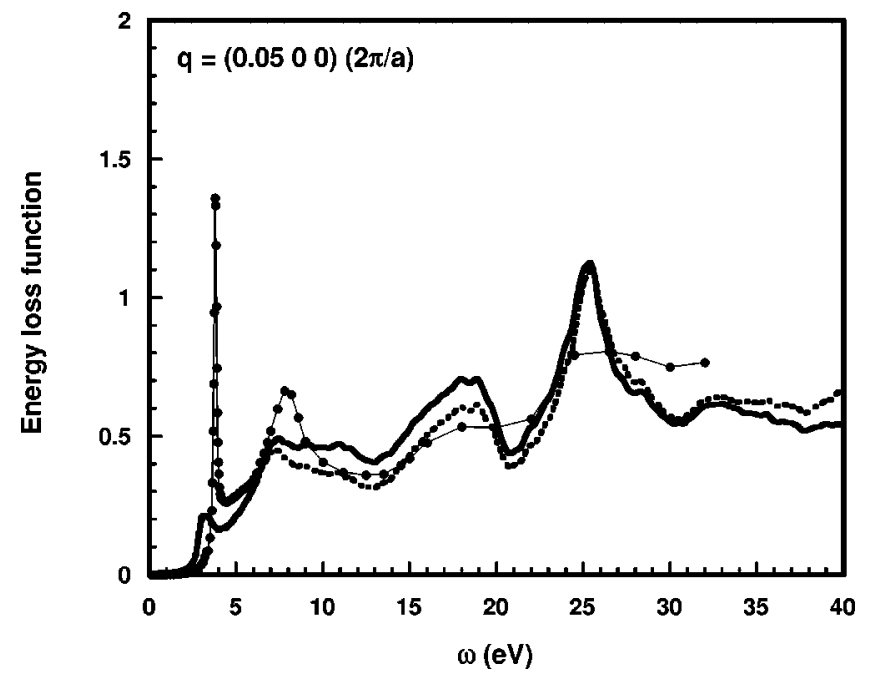

FIG. 4. Energy-loss spectrum of $\mathrm{Ag}$ for $q=(0.05,0,0)(2 \pi / a)$, and $|\mathbf{q}|=7.7 \times 10^{-2} \AA^{-1}$. The continuous line is the imaginary part of $\mathbf{G}=\mathbf{G}^{\prime}=\mathbf{0}$ element of the inverse dielectric matrix, namely, the energy-loss function $\operatorname{Im}\left[-\epsilon_{\mathbf{0 0}}^{-1}(\mathbf{q}, \omega)\right]$, whereas the dashed line corresponds to (the imaginary part of) one over the $\mathbf{G}=\mathbf{G}^{\prime}=\mathbf{0}$ element of the dielectric matrix $\operatorname{Im}\left[-1 / \epsilon_{\mathbf{0 0}}(\mathbf{q}, \omega)\right]$. Differences between them are due to crystal local-field effects. Dots are the experimental data from Ref. 47. As they were obtained from optical data and do not include crystal local-field corrections, they must be compared to the dashed line.

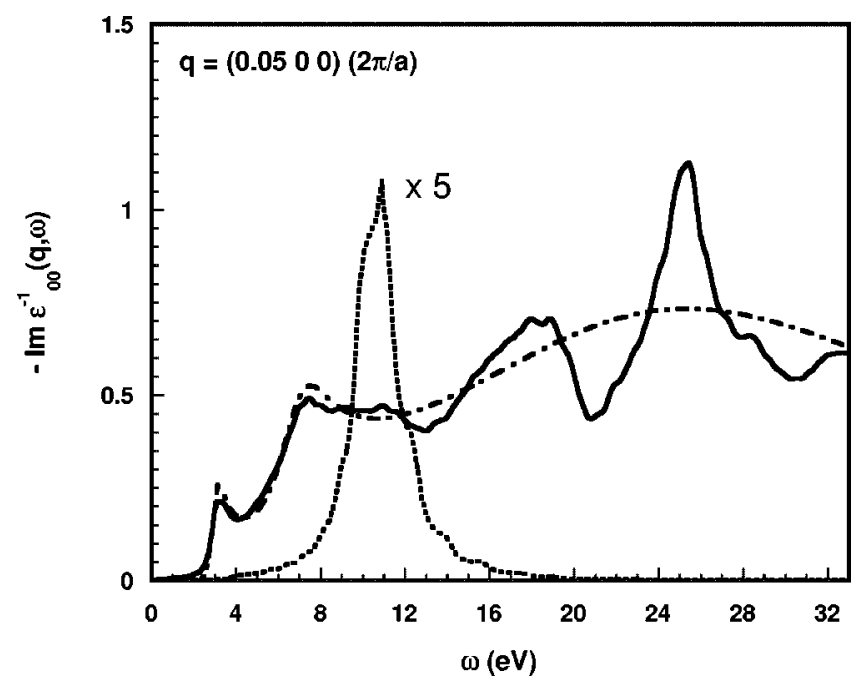

FIG. 5. Energy-loss spectrum of Ag for $q=(0.0500)(2 \pi / a)$, and $|\mathbf{q}|=7.7 \times 10^{-2} \AA^{-1}$. The continuous line is a calculation for real frequencies with $\eta=0.2 \mathrm{eV}$, cf. Eq. (13). The dot-dashed line, however, is obtained by analytically continuing an imaginary frequency calculation to the real axis, using a Padé approximant. As can be seen, the results obtained by analytical continuation cannot reproduce all the features in the spectrum obtained using a real $\omega$ (with a higher numerical damping $\eta$ ). The dashed line is a calculation of the loss function including the $4 d$ electrons of $\mathrm{Ag}$ in the frozen pseudocore that replaces the true ionic core. These electrons cannot be excited, thus showing the important role that they actually play in modifying the spectrum. Notice that the intensity of this peak is five times what is represented here.

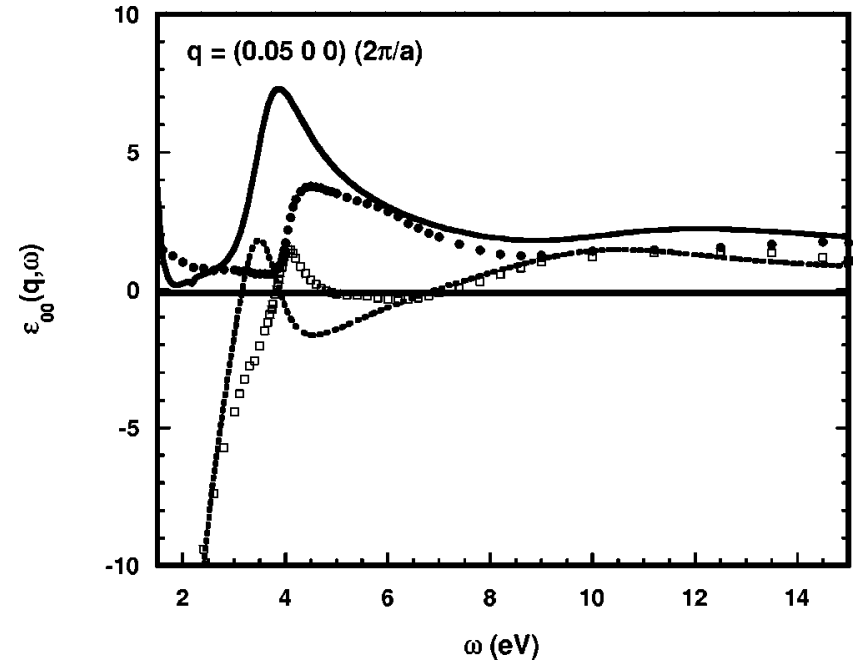

FIG. 6. Dielectric function $\epsilon_{\mathbf{0 0}}(\mathbf{q}, \omega)$ of $\mathrm{Ag}$ for $q=(0.05,0,0)$ $\times(2 \pi / a)$ and $|\mathbf{q}|=7.7 \times 10^{-2} \AA^{-1}$. The continuous line corresponds to the imaginary part as obtained by analytically continuing an imaginary frequency calculation, using a Padé approximant. The dashed line is the real part obtained by the same method. We checked that the results are consistent with a real frequency calculation (not showed here for clarity). Full circles (imaginary part) and open squares (real part) correspond to experimental data from Ref. 47.

$d$-like bands of Ag. Therefore, a more detailed description of correlation within the $d$-like bands seems to be required. ${ }^{46}$

Figure 4 presents our results for the energy-loss function for $\mathbf{q}=(0.05,0,0)(2 \pi / a)$ as obtained from the LDA band structure. Since our interest is focused on the plasmonic excitations, which occur for small $\mathbf{q}$, we need not exactly consider the $|\mathbf{q}|=0$ limit. Thus, for the small $\mathbf{q}$ considered, the theory developed in Sec. II still applies. The continuous line in this figure corresponds to $\operatorname{Im}\left[-\epsilon_{\mathbf{0 0}}^{-1}(\mathbf{q}, \omega)\right]$, whereas the dashed line corresponds to $\operatorname{Im}\left[-1 / \epsilon_{\mathbf{0 0}}(\mathbf{q}, \omega)\right]$, i.e., with and without including crystal local-field effects, respectively. As we already stated in Sec. II, crystal local-field effects are at most a correction $\sim 10 \%$ in the frequency range considered. Dots are the experimental data ${ }^{47}$ obtained by means of optical measurements (i.e., for $|\mathbf{q}| \rightarrow 0$ ), and should be compared with the dashed line. As a consequence of the LDA underestimate of $\omega_{T}$, the plasmon peak appears at a lower energy $\approx 3.1 \mathrm{eV}$ than observed experimentally ${ }^{10} \approx 3.8 \mathrm{eV}$. This peak also appears more damped both because of the high value of the numerical damping employed in the calculation and because of the LDA underestimate of $\omega_{T}$.

From Figs. 4 and 5 we can conclude the following:

(i) The Drude plasmon becomes so broadened by its coupling to interband transitions that we can no longer speak of a well-defined excitation. The high-energy tail of this broad feature around $8 \mathrm{eV}$ is more strongly affected by crystal local-field effects. This seems to indicate that these decay processes involve the excitation of an electron in a more localized state, probably lying deeper in the $d$-like band. The important role played by the $d$-like bands in destroying the Drude plasmon can be seen in Fig. 5, where the dashed line corresponds to a calculation of the energy-loss function including the ten $4 d$ electrons of atomic $\mathrm{Ag}$ in the frozen 
pseudocore. In this case, the Landau damping of the Drude plasmon is only due to transitions of type B, which have a much smaller spectral weight than those of type A. Furthermore, the plasma frequency is pushed upwards by this continuum of modes, which mainly lies below $\omega_{p}^{D}$. However, when electrons from the $d$-like bands are considered as active, the effect goes in the opposite direction thus shifting the maximum of the Drude peak to $\approx 7.5 \mathrm{eV}$. (ii) In agreement with the experiments, a double hump structure in the region between 15 and $30 \mathrm{eV}$ is found. This is a band-structure effect, which turns out to be a general feature in the noble metal systems. ${ }^{11}$ As can be seen in Fig. 5, although the calculation using a Padé approximant (dot-dashed line) gives a reliable description of the low-frequency part, it fails to reproduce the double-hump structure. This seems to be a rather general feature of analytic continuation by Padé approximants. Whenever the function to be continued presents two poles close to each other and having a large imaginary part, the values for the function on the real axis correspond to an average of the two poles, and a single broad peak shows up in the outcome of the analytical continuation. (iii) Altogether, the ab initio LDA calculations presented here provide a good description of the high-energy features (i.e., above $\left.\omega_{p}^{D}\right)$ of the energy-loss spectrum of Ag. These include the Landau damping of the Drude plasmon and the double-hump structure mentioned above. This could be expected on the basis that the spectral weight in the high-energy region of the spectrum comes, to a large extent, from the excitation of electrons to bands well above the Fermi level, which are expected to be more accurately reproduced by the LDA, as it is an approximation suitable for an homogeneous electron system. However, the LDA underestimate of the threshold energy is responsible for failing to reproduce the low-energy structure. This issue is investigated further below.

In Fig. 6, we show the results for the real and imaginary parts of $\epsilon_{\mathbf{0 0}}(\mathbf{q}, \omega)$ for $\mathbf{q}=(0.05,0,0)(2 \pi / a)$. The same remarks made above apply in this case as well. The experimental results, full circles for the imaginary part and open squares for the real part, are from Ref. 47. In this figure we can see more easily that the onset for the excitation of interband transitions not only occurs, at a lower energy, but that it is less sharp as well. As remarked in Sec. III this leads to a more damped plasmonic mode below the threshold.

In order to investigate the influence of the interband threshold energy on the existence of a threshold plasma resonance, we have performed a series of numerical "experiments." The motivation of these experiments is to understand why $\mathrm{Ag}$ presents a narrow plasma resonance in its energy-loss spectrum, while $\mathrm{Cu}$ (or $\mathrm{Au}$ ) does not, despite the similarity of their band structures. To modify the threshold energy $\omega_{T}$ without opening unphysical gaps in the band structure, we have scaled by a factor $\alpha$ the separation between the Fermi energy $\left(\varepsilon_{F}\right)$ and the LDA eigenvalues of the occupied states:

$$
\varepsilon_{n \mathbf{k}}^{\prime}=\varepsilon_{F}+\alpha\left(\varepsilon_{n \mathbf{k}}-\varepsilon_{F}\right)
$$

Using this new set of eigenvalues, we have calculated $\chi^{0}$, and hence obtained the energy-loss function. This rather $a d$ hoc procedure does not modify the wave functions accord-

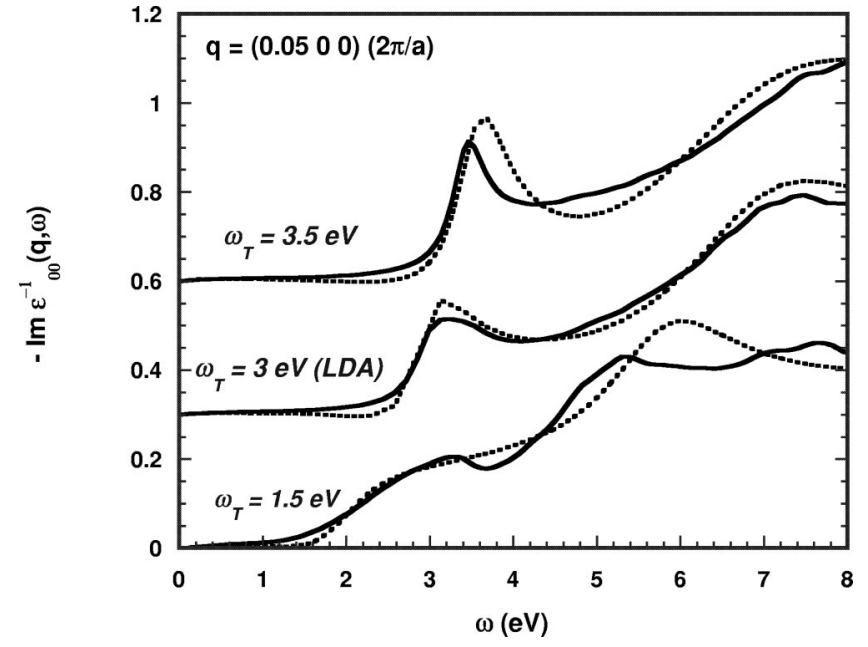

FIG. 7. Results of the scaling procedure described in Sec. IV. The continuous lines are the results as calculated for real $\omega$ with $\eta=0.2 \mathrm{eV}$, while the dashed lines are obtained by analytical continuation using Padé approximants. When the occupied bands are scaled so that the interband threshold energy increases, the threshold plasmon appears more defined. However, when the threshold energy is decreased the plasmon disappears from the energy loss spectrum.

ingly. Furthermore, it introduces a discontinuity of $\nabla_{\mathbf{k}} \varepsilon_{n \mathbf{k}}^{\prime}$ at the Fermi surface, so that it might lead to unphysical results. However, as shown in Fig. 7, this seems not to be the case, at least for $\omega \sim \omega_{T}$ and $\mathbf{q}$ finite. As predicted in Sec. II, the threshold plasmon peak is enhanced and shifts up in energy as $\omega_{T}$ increases. In this figure, continuous lines correspond to calculations with real frequencies for $\eta=0.2 \mathrm{eV}$, while dashed lines correspond to the analytic continuation using a Padé approximant from the results obtained on the imaginary frequency axis. Both calculations show the same tendency.

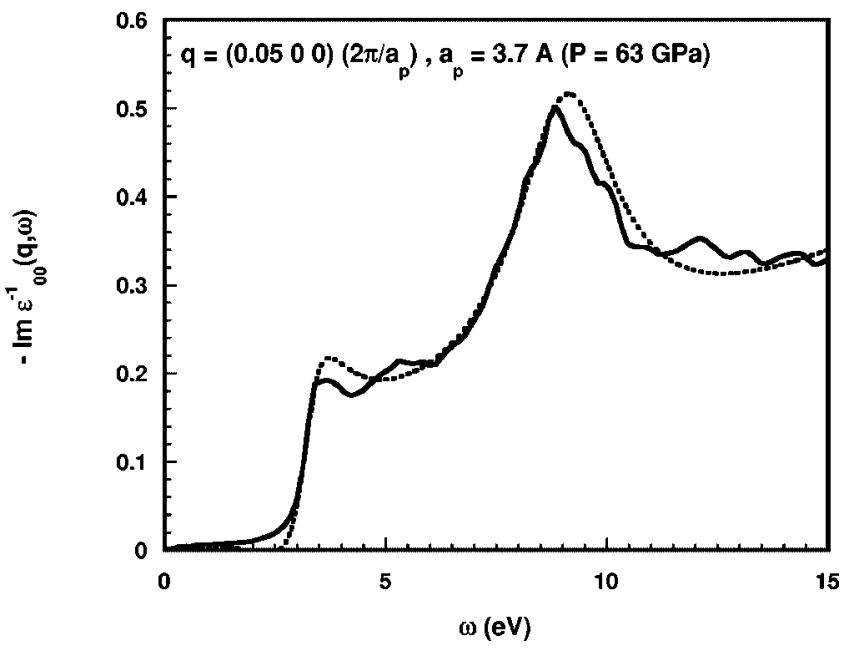

FIG. 8. Energy-loss spectrum of Ag for $q=(0.05,0,0)\left(2 \pi / a_{p}\right)$ $\left(|\mathbf{q}|=8.5 \times 10^{-2} \AA^{-1}\right)$ with $a_{p}=3.7 \AA$, which corresponds to an externally applied hydrostatic pressure of $63 \mathrm{GPa}$. The continuous line is a calculation for real frequencies with $\eta=0.2 \mathrm{eV}$, [cf. Eq. (13)]. The dashed line, however, is obtained by analytically continuing to the real axis an imaginary frequency calculation, using a Padé approximant. 
On the other hand, when the threshold energy is further decreased, from its LDA value $\approx 3.0 \mathrm{eV}$ to $\omega_{T} \approx 1.5 \mathrm{eV}$, the plasma resonance disappears and the spectrum becomes more "Cu like."

Finally, in Fig. 8 we show the energy-loss spectrum of Ag under an external pressure of $63 \mathrm{GPa}$. As in previous cases, the continuous line corresponds to a calculation using real frequencies while the dashed line is the result of an analytical continuation using a Padé approximant. Notice that in this case the lattice constant is $a_{p}=3.7 \AA$, so that now $q$ $=(0.05,0,0)\left(2 \pi / a_{p}\right)$, corresponds to a slightly larger momentum $\left(\left|\mathbf{q}_{p}\right|=8.5 \times 10^{-2} \AA^{-1}\right.$ vs $\left.|\mathbf{q}|=7.7 \times 10^{-2} \AA^{-1}\right)$.

Decreasing the lattice constant changes the width of the bands so that they disperse more rapidly with $\mathbf{k}$ (i.e., the band width becomes larger). Furthermore, electronic density also increases. This shifts the maximum previously at $\omega^{\max }$ $\approx 7.5 \mathrm{eV}$ to $\omega_{\text {press }}^{\max } \approx 8.8 \mathrm{eV}$. Since this maximum is related to the Drude plasmon, whose bare frequency scales with the lattice parameter as $a^{-3 / 2}$, we could understand the shift (neglecting the change in the optical mass) as due to the increase in the electronic density. Indeed, this argument works reasonably well as $\left(a_{p} / a\right)^{-3 / 2}=1.16$, and $\omega_{\text {press }}^{\max } / \omega^{\max }$ $\approx 1.17$. Moreover, as the bands become wider, especially those corresponding to excited states, more oscillator strength is transferred to higher energies. This decreases the density of modes at the Drude frequency, and thus the corresponding peak is now less broad. What is more, in this spectrum the threshold plasmon is no longer present. Again, this is due to the increased electronic density, which yields a higher value for the Drude plasma frequency so that Eq. (7) has no longer a solution close to the real axis. The behavior under pressure predicted here remains to be confirmed by experiments.

The existence of large band gaps in the band structure of Ag seems to point out that the threshold excitation observed in $\mathrm{Ag}$ corresponds to the zone boundary collective state (ZBCS) first described by Foo and Hopfield, ${ }^{23}$ and studied with great detail by Sturm and Oliveira in the case of $\mathrm{Al}^{, 24}$ $\mathrm{Na}^{25}$ and $\mathrm{Li}^{25,27}$ In these systems, the existence of a gap at the BZ boundary produces sharp variation of the density of modes, thus leading to an excitation of the kind described here. In the present case, however, the sharpness of the interband onset has a different origin, related to the presence of a set of $d$-like bands, with different symmetry compared to the unoccupied states in the conduction band. As a consequence, the appearance of the threshold plasmon does not depend on the direction of $\mathbf{q}$ as in the ZBCS case. ${ }^{48}$ Moreover, it seems difficult to understand the result of the numerical experiments shown in Figs. 7 and 8 in terms of a "standard" ZBCS. Finally, as predicted ${ }^{24,25}$ and also found in recent ab initio calculations, ${ }^{27}$ the spectral weight of a ZBCS increases with $|\mathbf{q}|$ along the direction where the band gap exists. However, Fig. 9 shows that the corresponding peak in $\mathrm{Ag}$ rapidly fades away as $|\mathbf{q}|$ is increased.

\section{CONCLUSIONS}

We have shown that a first-principles calculation of the energy-loss spectrum is able to reproduce, within the RPA, the high-energy structure in the spectrum of Ag. However, since the LDA underestimates the interband energy thresh-

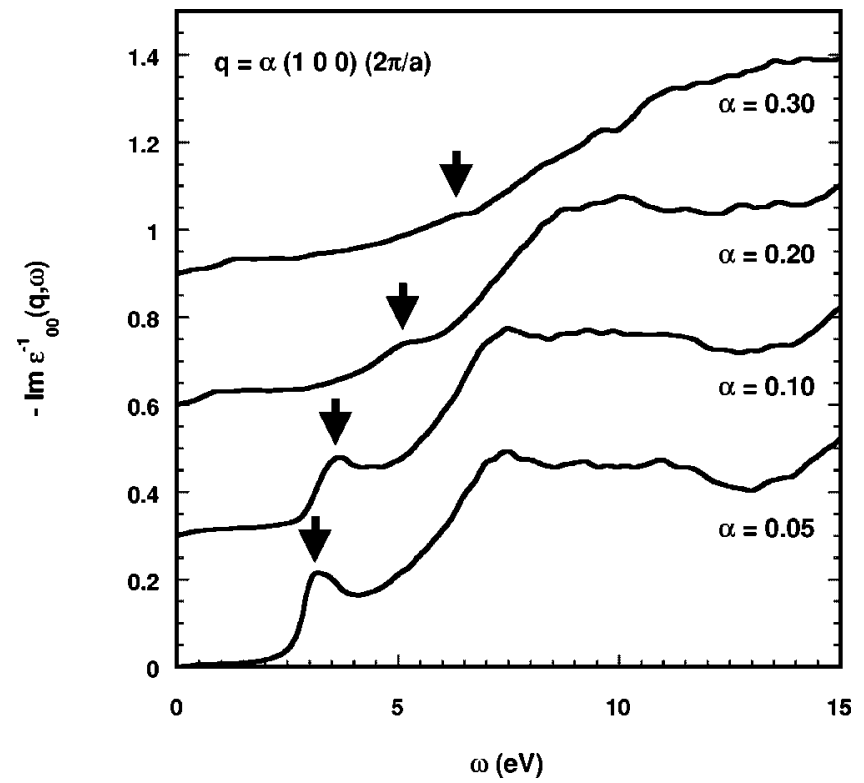

FIG. 9. Dispersion of the threshold plasmon in Ag along the $(1,0,0)$ direction. The peak rapidly disperses upward in energy, and damps down.

old, the agreement is not as good as for the high-energy region around $4 \mathrm{eV}$. For example, the plasma resonance observed experimentally at $3.8 \mathrm{eV}$ appears as a more damped peak at $\approx 3.1 \mathrm{eV}$. In our opinion, this underestimate may be related to a failure of the LDA to account for electron correlation effects that take place in the flat $d$-like bands of Ag. In fact, an ad hoc scaling of the occupied bands, so that the threshold energy is brought closer to the experimental value, gives rise to a better defined threshold plasmonic excitation. We have also shown the important role played by $d$-symmetry bands in determining the existence of plasma resonance at $3.8 \mathrm{eV}$, just below the interband excitation threshold. By using a simple model, it is shown that the appearance of this excitation can be related to a sharp interband onset and the relative values of the threshold energy and the Drude plasma frequency. Since it contains very little oscillator strength, it is very sensitive to impurities, defects, and changes in the band structure. Thus we have demonstrated, by performing numerical experiments, that its presence depends on the value of the threshold energy. Finally, we have also computed the energy-loss spectrum of Ag under pressure, and found that its features can also be understood in terms of the simple model presented here. We stress that the analysis carried out in this work is not specific to $\mathrm{Ag}$, and could be readily extended to other metallic compounds with fully occupied $d$-symmetry bands close to the Fermi level.

Note added in proof. After the manuscript was accepted, several works have been brought to our attention concerning the convergence of the analytic continuation method using Padé approximants. Although it has been recently pointed out ${ }^{49}$ that analytical continuation of $\epsilon_{\mathbf{G G}^{\prime}}(\mathbf{q}, i \nu)$ before inverting it gives better results, it has also been argued ${ }^{50}$ that the scheme used here and in Ref. 49, based on Thiele's reciprocal difference method, seems to be rather unstable with respect to error propagation. Moreover, as shown in Ref. 50, in order to obtain reliable results using Padé approximants a 
high numerical accuracy (requiring symbolic computation algorithms) is needed. These authors also demonstrate that the conventional criterion of stability with respect to increasing the number of input points is not reliable. In general, instability with respect to noise seems to be a rather common feature of the analytic continuation methods. ${ }^{51}$ As we compare the results obtained by analytical continuation with real frequency calculations, these instabilities do not affect the conclusions of this work.

\section{ACKNOWLEDGMENTS}

We thank N. Lorente, J. F. Dobson, and L. Hedin for fruitful discussions, and I. Campillo for many useful suggestions. This work was supported by the Basque Unibertsitate and Hezkuntza Saila, JCyL (VA28/99), and Iberdrola, S.A. M.A.C. would like to thank the Basque Government for financial support, and J.S.D. a grant from The University of Basque Country/Euskal Herriko Unibertsitatea. M.A.C. and J.S.D. wish to acknowledge the kind hospitality of the $D e$ partamento de Física Teórica in Valladolid.

*Present address: Department of Physics, Brown University, Providence, RI 02912-1843. Electronic address: miguel@baras.physics.brown.edu

${ }^{1}$ W. Ku and A.G. Equiluz, Phys. Rev. Lett. 82, 2350 (1999).

${ }^{2}$ A. vom Felde, J. Sprösser-Prou, and J. Fink, Phys. Rev. B 40, 10 181 (1989).

${ }^{3}$ A.A. Quong and A.G. Eguiluz, Phys. Rev. Lett. 70, 3955 (1993).

${ }^{4}$ F. Aryasetiawan and K. Karlsson, Phys. Rev. Lett. 73, 1679 (1994).

${ }^{5}$ A. Fleszar, R. Stumpf, and A.G. Eguiluz, Phys. Rev. B 55, 2068 (1997).

${ }^{6}$ N.E. Maddocks, R.W. Godby, and R.J. Needs, Europhys. Lett. 27, 681 (1994); Phys. Rev. B 49, 8502 (1994).

${ }^{7} \mathrm{H}$. Raether, Excitation of Plasmons and Interband Transitions by Electrons (Springer-Verlag, Berlin, 1980).

${ }^{8}$ W. Schülke, J.R. Schmitz, H. Schulte-Schrepping, and A. Kaprolat, Phys. Rev. B 52, 11721 (1995).

${ }^{9}$ F. Wooten, Optical Properties of Solids (Academic Press, New York 1972).

${ }^{10}$ H. Ehrenreich and H.R. Phillipp, Phys. Rev. 128, 1622 (1962).

${ }^{11}$ I. Campillo, A. Rubio, and J. M. Pitarke, Phys. Rev. B (to be published). (1999).

${ }^{12}$ C.B. Wilson, Proc. Phys. Soc. London 76, 481 (1960).

${ }^{13}$ K. Sturm, Adv. Phys. 31, 1 (1982).

${ }^{14}$ For an homogeneous electron gas in the RPA, a plasmon with a momentum less than the so-called Landau cutoff (Ref. 29) $q_{c}$ $\approx \omega_{p}^{c} / k_{F}$ cannot decay into an electron-hole pair. For $\mathrm{Ag}\left(r_{s}\right.$ $\left.=3.02 a_{0}\right), \quad q_{c}=0.98 \AA^{-1}$, and $q_{c}=1.04 \AA^{-1}$ for $\mathrm{Cu}$ (rs $\left.=2.67 a_{0}\right)$.

${ }^{15}$ E. Zaremba and K. Sturm, Phys. Rev. Lett. 55, 750 (1985).

${ }^{16}$ E.A. Taft and H.R. Phillipp, Phys. Rev. 121, 1100 (1961).

${ }^{17}$ B.R. Cooper, H. Ehrenreich, and H.R. Phillipp, Phys. Rev. 138, A494 (1965).

${ }^{18}$ F.M. Mueller and J.C. Phillips, Phys. Rev. 157, 600 (1967).

${ }^{19}$ G.A. Burdick, Phys. Rev. 129, 138 (1963).

${ }^{20}$ R.M. Morgan and D.W. Lynch, Phys. Rev. 172, 628 (1968).

${ }^{21}$ G.B. Irani, T. Huen, and F. Wooten, Phys. Rev. B 3, 2385 (1970).

${ }^{22}$ M. Urner-Wille and H. Raether, Phys. Lett. 58A, 265 (1976).

${ }^{23}$ E-N. Foo and J.J. Hopfield, Phys. Rev. 173, 635 (1968).
${ }^{24}$ K. Sturm and L.E. Oliveira, Phys. Rev. B 30, 4351 (1984).

${ }^{25}$ K. Sturm and L.E. Oliveira, Phys. Rev. B 40, 3672 (1989).

${ }^{26}$ A. Fleszar, A.A. Quong, and A.G. Eguiluz, Phys. Rev. Lett. 74, 590 (1995).

${ }^{27}$ K. Karlsson and F. Aryasetiawan, Phys. Rev. B 52, 4823 (1995).

${ }^{28}$ N. W. Ashcroft and N. D. Mermin, Solid State Physics (Saunders College Press, Philadelphia 1976).

${ }^{29}$ D. Pines, Elementary Excitations in Solids (Benjamin, New York, 1965).

${ }^{30}$ J.W. Gadzuk, Phys. Rev. B 24, 1651 (1981).

${ }^{31}$ G.D. Mahan, Many-Particle Physics (Plenum, New York, 1990).

${ }^{32}$ U. Fano, Phys. Rev. 124, 1866 (1961).

${ }^{33}$ P.W. Anderson, Phys. Rev. 124, 41 (1961).

${ }^{34}$ S. Engelsberg and J.R. Schrieffer, Phys. Rev. 131, 993 (1963).

${ }^{35}$ A. Liebsch, Phys. Rev. Lett. 71, 145 (1993).

${ }^{36}$ Ll. Serra and A. Rubio, Phys. Rev. Lett. 78, 1428 (1997).

${ }^{37}$ D. Pines and P. Nozières, The Theory of Quantum Liquids (Benjamin, New York 1966), Vol. 1.

${ }^{38}$ E.K.U. Gross, J.F. Dobson, and M. Petersilka, in Density Functional Theory II, edited by R.F. Nalewajski, Topics in Current Chemistry Vol. 181 (Springer, Berlin, 1996), p. 81, and references therein.

${ }^{39}$ W. Kohn and L.J. Sham, Phys. Rev. 140, A1133 (1965).

${ }^{40}$ D.M. Ceperley and B.J. Alder, Phys. Rev. Lett. 45, 1196 (1980); as parametrized by J.P. Perdew and A. Zunger, Phys. Rev. B 23, 5048 (1981).

${ }^{41}$ N. Troullier and J.L. Martins, Phys. Rev. B 43, 1993 (1991). It requires $\approx 1400$ plane waves per Bloch state up to a cutoff energy of $\approx 1.1 \mathrm{keV}$ in order to obtain a well-converged groundstate energy (less than $0.01 \mathrm{eV}$ ), as well as single-electron energies and orbitals up to $\approx 100 \mathrm{eV}$. The lattice constant has been set to the experimental value of the fcc crystal structure of Ag, namely, $a=4.09 \AA$. We have also solved the Kohn-Sham equations with $a_{p}=3.70 \AA$, which corresponds to an externally applied hydrostatic pressure of $63 \mathrm{GPa}$, assuming that Ag maintains a fcc structure under such a high pressure.

${ }^{42}$ To evaluate $\chi^{0}$ using Eq. (13), we have cut off the sum over $n$ and $m$ using bands up to an energy of $\approx 100 \mathrm{eV}$. The integration over the first BZ has been performed using a $20 \times 20 \times 20$ Monkhorst-Pack mesh [H.J. Monkhorst and J.D. Pack, Phys. Rev. B 13, 5188 (1976)], in the irreducible BZ, which allows us to deal effectively with the topology of the Fermi surface of Ag. Indeed, this is a very dense mesh, and the calculation of $\chi^{0}$ becomes certainly time and memory consuming. However, if one is to resolve the very narrow plasma resonance that $\mathrm{Ag}$ presents near the interband excitation threshold, it is then necessary to work with such a fine mesh. To calculate the dielectric matrix we have used a cutoff of $41 \mathrm{eV}$ in $\mathbf{G}$ and $\mathbf{G}^{\prime}$, which amounts to using $59 \times 59$ matrices.

${ }^{43}$ K.-H. Lee and K.J. Chang, Phys. Rev. B 54, R8285 (1996).

${ }^{44}$ W.H. Press, S.A. Teukolsky, W.T. Vetterling, and B.P. Flannery, Numerical Recipes (Cambridge University Press, Cambridge 1992). Using this algorithm, we work with the ratio of the numerators and denominators obtained when the degrees of the numerator and denominator are increased by 1 . This allows us to avoid numerical overflow and round off problems which appear when a large number of points is used to fit the Padé approximant.

${ }^{45}$ We have used up to 1600 points over up to $110 \mathrm{eV}$ in $\nu$.

${ }^{46}$ J.S. Dolado, M.A. Cazalilla, A. Rubio, and P. M. Echenique (unpublished). 
${ }^{47}$ E.D. Palik, Handbook of Optical Constants of Solids (Academic Press, New York, 1985).

${ }^{48} \mathrm{We}$ have checked the presence of the plasmonic excitation in various directions [as for instance $(1,1,2)]$, in addition to the $(1,0,0)$ shown here.
${ }^{49}$ Young-Cu Jin and K.J. Chang, Phys. Rev. B 59, 14841 (1999).

${ }^{50}$ K.S.D. Beach, R.J. Gooding, and F. Marsiglio, Phys. Rev. B 61, 5147 (2000)

${ }^{51}$ E. Gallicchio, S. A. Egorov, and B. J. Berne, J. Chem. Phys. 109, 7745 (1998). 